\title{
Utilization of Mechanical Quadrature in Silicon MEMS Vibratory Gyroscope to Increase and Expand the Long Term In-Run Bias Stability
}

\author{
Sergei A. Zotov, Brenton R. Simon, Gunjana Sharma, Alexander A. Trusov, Andrei M. Shkel \\ MicroSystems Laboratory, University of California, Irvine, CA, USA \\ Email: \{szotov, brsimon, gunjanas, atrusov, ashkel\}@uci.edu
}

\begin{abstract}
We report a new approach for improvement of the long term in-run bias stability of Coriolis vibratory gyroscopes. The approach is based on utilization of the mechanical quadrature error in gyroscopes to compensate for variation in system parameters. The proposed approach was validated by a silicon Quadruple Mass Gyroscope, with the natural frequency of $3 \mathrm{kHz}$, frequency mismatch of $<0.5 \mathrm{~Hz}$, and isotropic quality factor of 950, packaged without getter. The algorithm is described and experimentally demonstrated in this paper, showing a bias stability of $0.1 \mathrm{deg} / \mathrm{hr}$ after 300 seconds, and importantly, retaining that value for over 3 hours of the integration time.
\end{abstract}

\section{INTRODUCTION}

The level of long term instability of bias and scale factor are key characteristics defining the utilization of gyroscopes in many practical applications, including navigation, positioning, and targeting systems. Thermal sensitivity of system's components accounts for the first order drift mechanisms of gyroscopes.

Thermal variations are typically identified and calibrated by utilizing the linear dependence of the gyroscope bias on the drive-mode resonant frequency [1], [2]. This approach, however, only captures the thermal drift of the mechanical parameters, as reflected in the mechanical resonant frequency of the device. The thermal sensitivity of other system components, such as electronic gains, remain unobservable.

The thermal drifts of the electronic components for the drive-mode can be avoided by using the Side Band Ratio (SBR) method [3], [4]. The SBR algorithm takes advantage of the non-linearity of parallel-plate sense capacitors, allowing a direct measurement of large mechanical amplitudes. However, this method can only be applied to the drive-mode, leaving the drift in sense-mode electronic components unobservable. Thus, there is a need to find a method of capturing the thermal drift of sense mode pick off components, thus increasing longterm stability of in-run bias and scale factor.

We introduce an algorithm that incorporates the quadrature signal in in-run calibration of bias. Quadrature is typically considered a parasitic signal in gyroscopes which distorts the rotation-induced response of the sensor. We show in this paper

This material is based upon work supported by DARPA grant N66001-12C-4035 (Program Manager Dr. Robert Lutwak.)

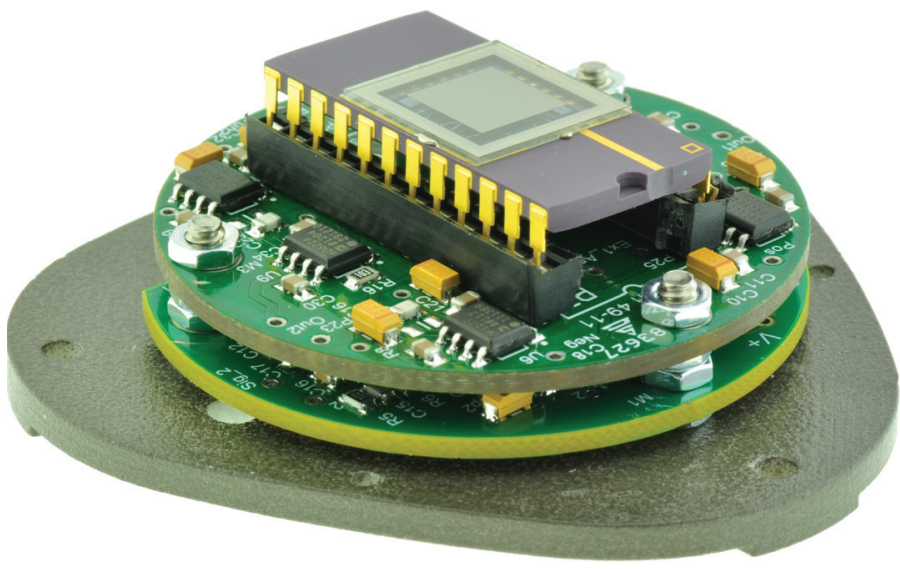

Fig. 1. Photograph of a device used for algorithm demonstration (quadruple mass gyroscope, fabricated in-house without getter material, and mounted on a two stage PCB)

that the quadrature signal can be utilized for compensation of thermal drift in sense mode detection system. We demonstrated that variations in the quadrature signal can be used to compensate for the parasitics-induced rotation response, thus minimizing the influence of thermal drift in electronics.

In this paper, we describe and experimentally demonstrate the following calibration methods: a temperature self-sensing algorithm utilizing the drive-mode resonance frequency for calibration of thermal drift in the mechanical parameters of the system, a sideband-ratio approach for direct detection of mechanical drive-mode amplitude, and a new approach for compensation of thermal drift in the sense-mode pick off system by utilizing mechanical quadrature. By using all three methods of calibration, the highest level of long term in-run bias stability is achieved.

\section{EXPERIMENTAL SETUP}

A silicon Quadruple Mass Gyroscope (QMG) [5], [6] was used for a proof of concept. The device comprises four tines, each coupled with external levers to force the anti-phase resonance. Unlike previous published designs [5], internal levering structures were implemented, stiffening the remaining spurious vibratory modes and allowing the anti-phase resonance to be 


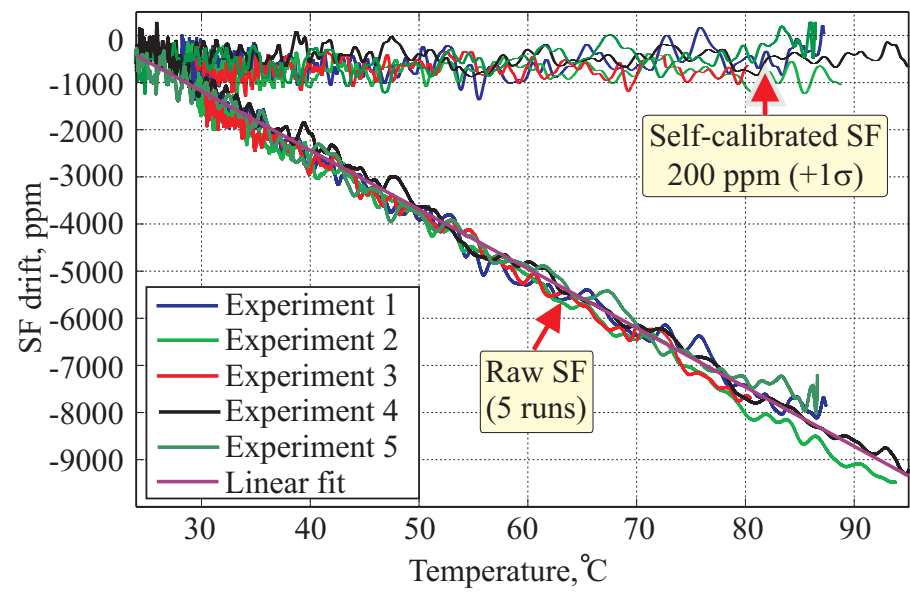

Fig. 2. Raw and compensated scale factor over dynamic temperature range over $60^{\circ} \mathrm{C}$, demonstrating residual drift of scale factor of the level $200 \mathrm{ppm}$.

the lowest frequency mode, reducing the influence of external acceleration [7].

Both the physical structure and interface electrodes were completely symmetric. The design used two sets of differential parallel plate electrodes attached to each mass and along each axis. The devices were fabricated using an in-house siliconon-insulator fabrication process, with device layer thickness of 100 microns, and sealed under moderate vacuum [8]. A vacuum-packaged QMG is mounted to a printed circuit board, Figure 1. In this paper, all results were obtained using a QMG with the following mechanical parameters: natural frequency of $3050 \mathrm{~Hz}$, mechanical frequency mismatch of less than 0.5 $\mathrm{Hz}$, and isotopic Q-factor of 950 (packaged without getter).

\section{TEMPERATURE SELF-SENSING}

The temperature self-sensing technique for capturing the thermal drift of the gyroscope's mechanical parameters is based on the continuous detection of the drive-mode operational frequency of the gyroscope. The frequency drift information was used for calibration of bias and scale factor. This technique was originally developed and used for the hemispherical gyroscopes by Northrop Grumman [1], and recently was demonstrated using a MEMS gyroscope [2].

As was shown in [2], this method is effective at achieving long term in-run bias stability of MEMS gyroscopes. In addition to the validation of this benefit, temperature-dependent scale-factor drifts are also shown to be reduced through temperature self-sensing. This was done through the use of an SOI Quadruple Mass Gyroscope (QMG), Figure 1, which was characterized on a precision-controlled dual-axis Ideal Aerosmith 2102 rate table. The temperature of the gyroscope was changed from $90^{\circ} \mathrm{C}$ to $30^{\circ} \mathrm{C}$ for 90 minutes. During this time, scale factor of the gyroscope was constantly collected. A linear relation was derived between the measured scale factor and temperature (provided by drive-mode frequency) of the gyroscope. The calibration coefficient was calculated by using this correlation, and the experiment was repeated five times with the fixed thermal model coefficient.

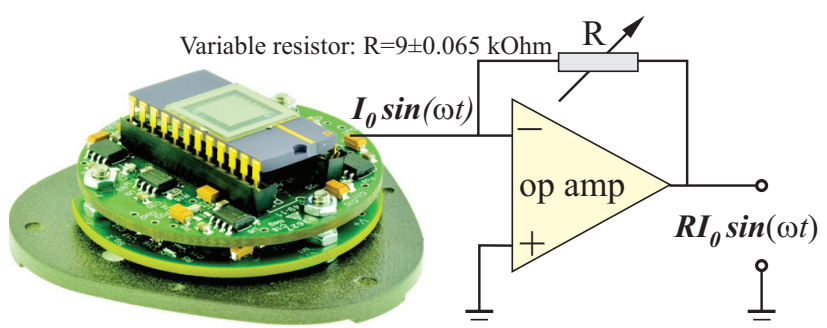

(a) Simplified experimental schematic used to quantity the SBR calibration technique, allowing a variable pick-off gain.



(b) Conventional and SBR detection methods. SBR algorithm shown inherent self-calibration against drift of the detection system gain by $1.4 \%$ with residual fluctuations of $96 \mathrm{ppm}(+1 \sigma)$.

Fig. 3. The SBR algorithm takes advantage of the non-linearity of parallelplates, showing inherent self-calibration against gain drifts of electronics.

Figure 2 shows the raw data and residuals of the scale factor drift after compensation. The analysis of this data revealed that the scale factor drift before compensation was up to 9,000 ppm; after compensation, the drift is reduced to $200 \mathrm{ppm}(+1 \sigma)$. The approach demonstrates a significant improvement in precision of the gyroscope's scale factor, throughout a wide temperature range of $60^{\circ} \mathrm{C}$.

\section{Side BAnd Ratio Detection}

In this section we experimentally demonstrate that the Side Band Ratio (SBR) method [3] allows for the direct detection of mechanical amplitude, which is independent of drift within the parameters of detection electronics.

The SBR approach is used for a direct detection of the drivemode mechanical amplitude of the gyroscope. The approach is based on non-linear capacitive detection, which is shown to be robust to variations of critical parameters, such as nominal capacitance, frequency, amplitude of the probing voltage, and amplifier gains [3], [4]. The method constructively utilizes the inherent nonlinearity of parallel-plate sense capacitors to measure sinusoidal motion. For parallel-plate detection, multiple harmonics exist and contain information about the mechanical motion [4]. The SBR method extracts the motion of the drive mode amplitude from the ratio of multiple nonlinear sidebands, theoretically creating a signal inherently selfcalibrated and robust against system drifts, such as drift of pick-off electronics.

To experimentally investigate this hypothesis, the gyroscope electronics was designed with a variable resistor of value 

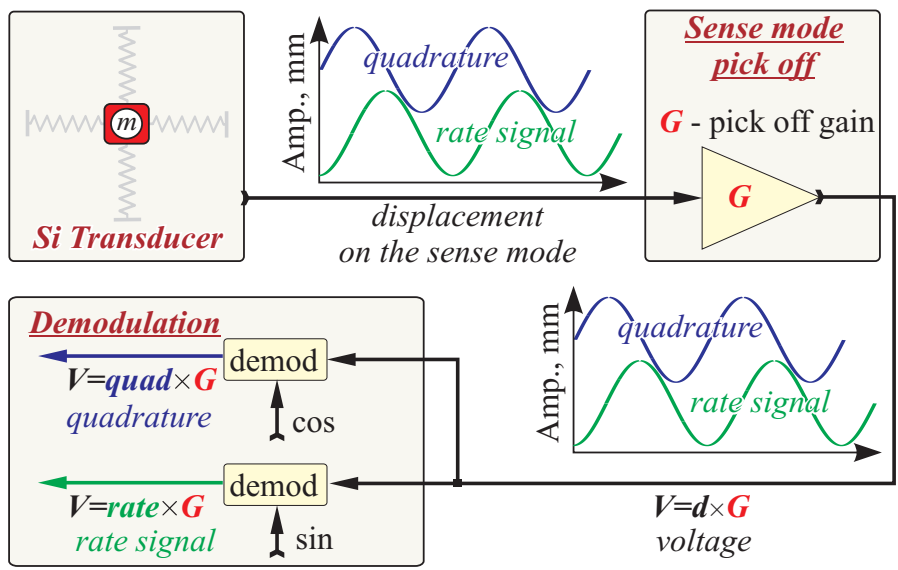

Fig. 4. Conceptual schematic of transducer and electronic drift, showing an identical pick-off gain, $G$, for both the rate signal and quadrature. By calibrating the rate signal against quadrature, changes in the rate signal due to amplifier drift are reduced.

$9 \pm 0.065 \mathrm{kOhm}( \pm 0.7 \%)$. This resistor controls the gain of the read-out electronics, which represents changes in pickoff gain due to temperature, and other environmental factors, Figure 3(a). The gyroscope drive-mode was then actuated by applying a constant DC voltage and a constant amplitude AC voltage. The output amplitude was measured using two different methods: traditional motional current detection and the SBR technique. The traditional method varied widely due to changes in resistance, on the order of $1.4 \%(14,000 \mathrm{ppm})$, while the SBR method deviated by only $96 \mathrm{ppm}(+1 \sigma)$, Figure 3(b). As data shows, the motional amplitude is measured precisely with inherent self-calibration against drifts of the system parameters, thus providing a highly stable method for direct observation of the mechanical drive-mode amplitude.

\section{QUADRATURE COMPENSATION}

Quadrature is typically considered a parasitic signal in gyroscopes which distorts the rotation-induced response of the sensor. In this section we experimentally demonstrated that the quadrature signal can be in-fact utilized for compensation of thermally induced drift in the sense mode detection system. This is accomplished by observing that the rotation-induced response and quadrature are two components of one physical signal, that feeds through the pick-off sense electronics. The amplitudes of both components (rotation-induced response and quadrature) change proportionally and in response to variations in mechanical structure and parameters of the control electronics, Figure 4. Using this observation, variations in the quadrature signal can be used to compensate for the rotationinduced response, thus minimizing the influence of thermal drift in electronics.

To validate this approach, the gyroscope's zero-rate output and quadrature signals were collected for 12 hours. During this period of time, temperature drifted in the range of $1.5^{\circ} \mathrm{C}$, and was monitored by use of the temperature self-sensing method described in section III.



Fig. 5. Algorithm for gyroscope self-calibration using drive-mode frequency and qudrature for bias drifts cancellation.

A measured 99.85 percent correlation between the quadrature and the gyroscope bias confirmed that the changes in the rate signal due to pick off gain drift can be effectively reduced, Figure 4. Uncompensated gyroscope output revealed a $14 \% \mathrm{hr}$ $(+1 \sigma)$ bias uncertainty over a dynamic temperature range of $1.5^{\circ} \mathrm{C}$. The calibration utilizing both the drive-mode frequency and quadrature of the device removed the bias drift of the device, yielding a $0.2 \% \mathrm{hr}(+1 \sigma)$ bias uncertainty, Figure 5 .

\section{Full COMPENSATION}

In this section we describe the simultaneous implementation of a combination of three calibration methods: frequency temperature self-sensing, sideband-ratio detection, and quadrature compensation. This combination allows for the full calibration of drift in the gyroscope, which includes drift of the mechanical transducer, as well as drift of drive-mode and sense-mode detection electronics.

To illustrate this approach, a vacuum-packaged QMG was mounted to a printed circuit board, Figure 3(b), containing a signal detection electronics. Front-end amplification of the output signals was done using two transimpedance and instrumentation amplifiers for each drive-mode and sensemode. The processing was implemented on a real-time DSP field-programmable gate array lock-in amplifier HF2 from Zurich Instruments. Separation of the useful signal from the feedthrough signal was accomplished using Electromechanical Amplitude Modulation (EAM). Instead of using a DC bias, an AC carrier voltage of $2.5 \mathrm{~V}$ at $52 \mathrm{kHz}$ was applied to the proof mass, resulting in the amplitude modulation of the motional signal [9]. The signals of both the drive- and sense-modes were fed to phase-locked loops (PLLs), which provided a continual operation of the gyroscope at mechanical resonance. The frequency provided by the PLL was used as an input to the temperature self-sensing algorithm, [2], and utilized for the compensation of temperature-induced bias drifts of the silicon transducer, Figure 6. Through temperature characterization of the gyroscope, the Temperature Coefficient of Frequency (TCF) was derived to be $-20 \mathrm{ppm} /{ }^{\circ} \mathrm{C}$. Combined with the $0.08 \mathrm{ppm}$ frequency stability of the device, a temperature selfsensing accuracy of $4^{\circ} \mathrm{mC}$ was demonstrated. 


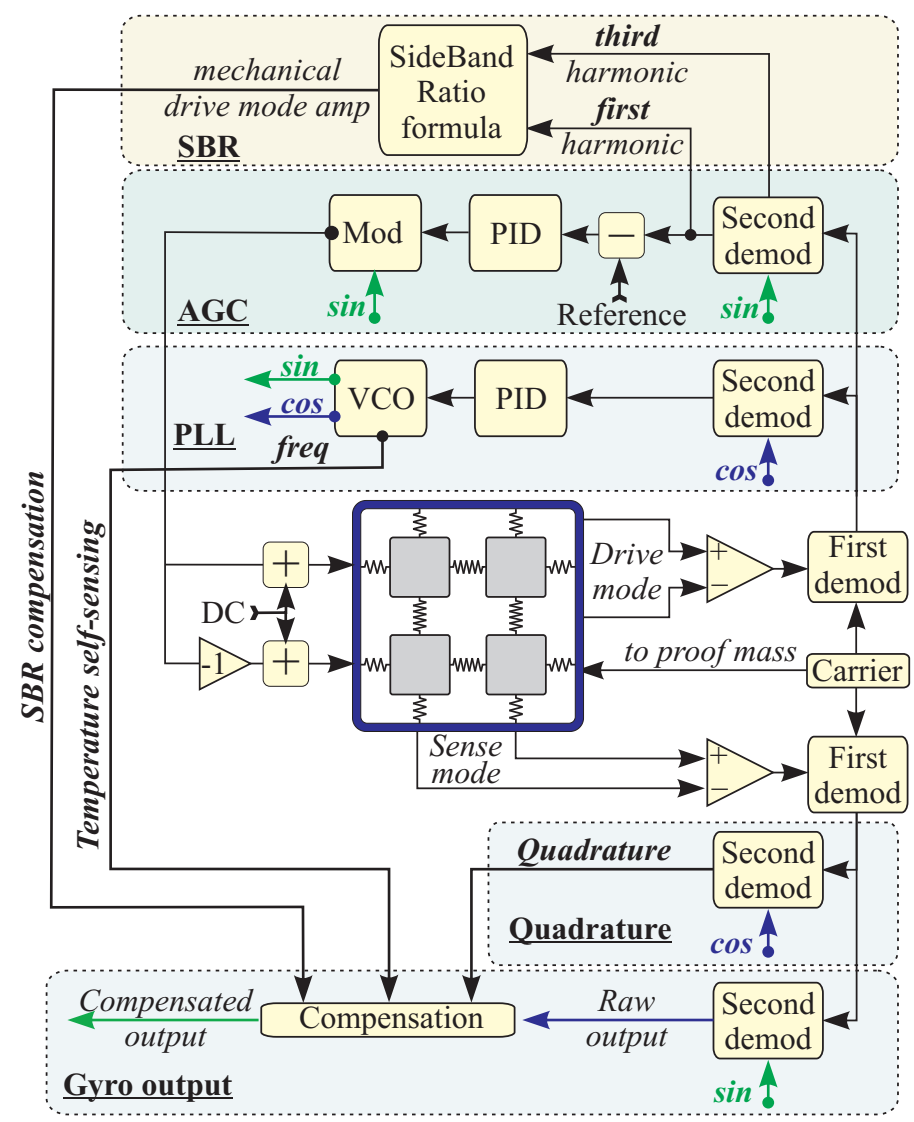

Fig. 6. Signal processing for self-compensation of long term in-run gyroscope bias by using simultaneously three calibration methods: temperature selfsensing, sideband-ratio detection, quadrature compensation.

The drive-mode amplitude of the gyroscope was stabilized through the use of Automatic Gain Control (AGC), which traditionally utilizes the first harmonic of the drive-mode detected signal. In addition to the first harmonic, higher order harmonics are produced by the demodulation of the signal. These higher order harmonics are influenced by the mechanical amplitude of the transducer, and therefore can be used to isolate the parasitic information from the useful pickoff signal. By separating these two factors, a steady mechanical amplitude can be maintained, as well as accomplishing the compensation of the drift in the drive-mode electronics.

Compensation of the sense-mode electronic drift was extracted from the quadrature signal of the sensor by taking advantage of the identical pick-off gains, $G$, for both the rotation-induced response and quadrature.

Allan deviation analysis of the raw gyroscope output demonstrated an angle random walk of $0.02 \% / \sqrt{h r}$, bias instability of $0.3^{\circ} \mathrm{hr}$, and a +1 slope temperature ramp at time constants above 100 seconds, Figure 7, (curve 1). Implementation of the temperature self-sensing approach alone, along with the SBR method, allows for the partial compensation of gyroscope thermal drift, Figure 7, curve 2 and curve 3.

In contrast, the fully calibrated gyroscope, which utilizes all three calibration methods, removed the +1 slope, revealing

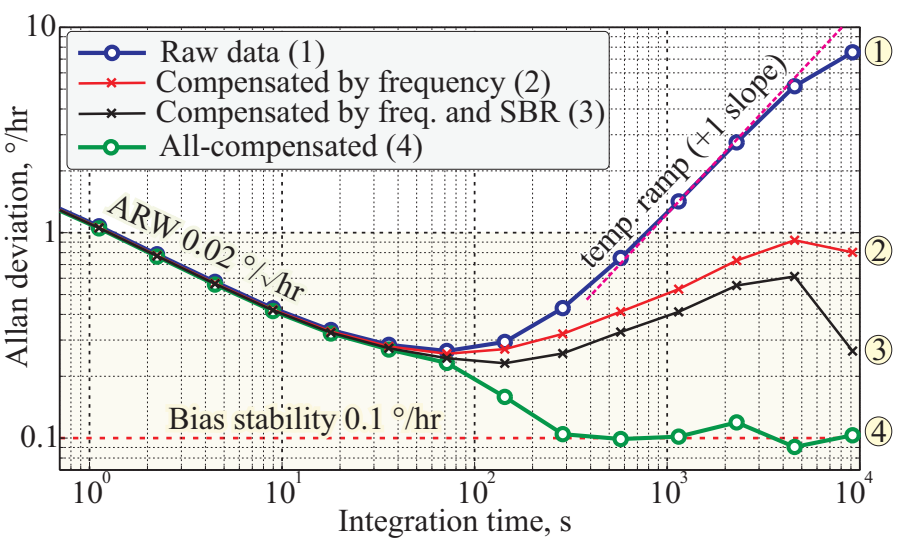

Fig. 7. Measured Allan deviation of gyroscope's zero rate output. Selfcalibration removes temperature ramp and achieves a $0.1^{\circ} / \mathrm{hr}$ bias instability for up to 10,000 seconds (3 hours).

the bias uncertainty of $0.1^{\circ} / \mathrm{hr}$ after 300 seconds, and retained this value for over 3 hours, Figure 7 , curve 4, limited only by the duration of experiment.

\section{CONCLUSION}

In this paper we report a new approach for increasing and expanding the long-term in-run bias stability of silicon MEMS vibratory gyroscope. This approach is based on utilization of gyroscope quadrature error for the compensation of parameter variation in the sense-mode detection system. Implementation of the proposed approach, along with side-band ratio and temperature self-sensing calibration methods, revealed a temperature-immune gyroscope output with long-term in-run bias uncertainty of $0.1^{\circ} / \mathrm{hr}$ after 300 seconds, while retaining this value for over 3 hours.

\section{REFERENCES}

[1] D.M. Rozelle, "The hemispherical resonator gyro: From wineglass to the planets," in Proc. 19th AAS/AIAA Space Flight Mechanics Meeting, Feb. 2009, pp. 1157-1178.

[2] I.P. Prikhodko, A.A. Trusov, A.M. Shkel, ”Achieving Long-Term Bias Stability in High-Q Inertial MEMS by Temperature Self-Sensing with a 0.5 Millicelcius Precision,” Solid-State Sensors, Actuators, and Microsystems Workshop 2012, Hilton Head Island, South Carolina, USA, June, 2012.

[3] A.A. Trusov, A.M. Shkel, "Apparatus and method using capacitive detection with inherent self-calibration," U.S. Patent 8,094,841.

[4] A.A. Trusov and A.M. Shkel, "A Novel Capacitive Detection Scheme with Inherent Self-Calibration," IEEE/ASME Journal of Microelectromechanical Systems, Vol. 16, No. 6, pp. 1324-1333, December 2007.

[5] A.A. Trusov, I.P. Prikhodko, S.A. Zotov, A.R. Schofield, and A.M. Shkel, "Ultra-high $Q$ silicon gyroscopes with interchangeable rate and whole angle modes of operation,” in Proc. IEEE Sensors, 2010, pp. 864-867.

[6] A. A. Trusov, A. R. Schofield, and A. M. Shkel, "Micromachined tuning fork gyroscopes with ultra-high sensitivity and shock rejection," U.S. Patent 20100313 657, Dec. 16, 2010.

[7] B.R. Simon, A.A. Trusov, A.M. Shkel, "Anti-Phase Mode Isolation in Tuning-Fork MEMS using a Lever Coupling Design,” IEEE Sensors 2012 Conference, Taipei, Taiwan, October 28 31, 2012.

[8] I.P. Prikhodko, B.R. Simon, G. Sharma, S.A. Zotov, A.A. Trusov, and A.M. Shkel, "High and Moderate Level Vacuum Packaging of Vibratory MEMS," 46-th International Symposium on Microelectronics (IMAPS 2013), Orlando, FL, USA, September 30 - October 3, 2013.

[9] A.A. Trusov and A.M. Shkel, "Capacitive detection in resonant MEMS with arbitrary amplitude of motion," J. Micromech. Microeng., vol. 17, no. 8 , pp. $1583-1592$, Jul. 2007 